\title{
DIVIDED DIFFERENCE OPERATORS ON POLYTOPES
}

\author{
VALENTINA KIRITCHENKO
}

\begin{abstract}
We define convex-geometric counterparts of divided difference (or Demazure) operators from the Schubert calculus and representation theory. These operators are used to construct inductively polytopes that capture Demazure characters of representations of reductive groups. In particular, Gelfand-Zetlin polytopes and twisted cubes of Grossberg-Karshon are obtained in a uniform way.
\end{abstract}

\section{INTRODUCTION}

Polytopes play a prominent role in representation theory and algebraic geometry. In algebraic geometry, there are Okounkov convex bodies introduced by KavehKhovanskii and Lazarsfeld-Mustata (see [KKh] for the references). These convex bodies turn out to be polytopes in many important cases (e.g. for spherical varieties). In representation theory, there are string polytopes introduced by BerensteinZelevinsky and Littelmann [BZ, L. String polytopes are associated with the irreducible representations of a reductive group $G$, namely, the integer points inside and at the boundary of a string polytope parameterize a canonical basis in the corresponding representation. A classical example of a string polytope for $G=G L_{n}$ is a Gelfand-Zetlin polytope.

There is a close relationship between string polytopes and Okounkov bodies. String polytopes were identified with Okounkov polytopes of flag varieties for a certain choice of a geometric valuation $[\mathrm{K}]$ and were also used in [KKh to give a more explicit description of Okounkov bodies associated with actions of $G$ on algebraic varieties. Natural generalizations of string polytopes are Okounkov polytopes of Bott-Samelson resolutions of Schubert varieties for various geometric valuations (an example of such a polytope is computed in [Anderson]).

In this paper, we introduce an elementary convex-geometric construction that yields polytopes with the same properties as string polytopes and Okounkov polytopes of Bott-Samelson resolutions. Namely, exponential sums over the integer points inside these polytopes yield Demazure characters. We start from a single point and apply a sequence of simple convex-geometric operators that mimic the

Key words and phrases. Gelfand-Zetlin polytope, divided difference operator, Demazure character.

The author was supported by Dynasty foundation, AG Laboratory NRU HSE, MESRF grants ag. 11.G34.31.0023, MK-983.2013.1 and by RFBR grants 12-01-31429-mol-a, 12-01-33101-mola-ved. This study was carried out within "The National Research University Higher School of Economics' Academic Fund Program in 2013-2014, research grant No. 12-01-0194". 
well-known divided difference or Demazure operators from the Schubert calculus and representation theory. Convex-geometric Demazure operators act on convex polytopes and take a polytope to a polytope of dimension one greater. In particular, classical Gelfand-Zetlin polytopes can be obtained in this way (see Section 3.2). More generally, these operators act on convex chains. The latter were defined and studied in [PKh] and used in [PKh2] to prove a convex-geometric variant of the Riemann-Roch theorem.

When $G=G L_{n}$, convex-geometric Demazure operators were implicitly used in [KST] to calculate Demazure characters of Schubert varieties in terms of the exponential sums over unions of faces of Gelfand-Zetlin polytopes and to represent Schubert cycles by unions of faces. A motivation for the present paper is to create a general framework for extending results of [KST] on Schubert calculus from type $A$ to arbitrary reductive groups. In particular, convex-geometric divided difference operators allow one to use in all types a geometric version of mitosis (mitosis on parallelepipeds) developed in [KST, Section 6]. This might help to find an analog of mitosis of $[\mathrm{KnM}]$ in other types.

Another motivation is to give a tool for describing inductively Okounkov polytopes of Bott-Samelson resolutions. We describe polytopes that conjecturally coincide with Okounkov polytopes of Bott-Samelson resolutions for a natural choice of a geometric valuation (see Conjecture 4.1). Another application is an inductive description of Newton-Okounkov polytopes for line bundles on Bott towers (in particular, on toric degenerations of Bott-Samelson resolutions) that were first described by Grossberg and Karshon [GK] (see Section 4.1 and Remark 4.6).

This paper is organized as follows. In Section 2, we give background on convex chains and define convex-geometric divided difference operators. In Section 3, we relate these operators with Demazure characters and their generalizations. In Section 4, we outline possible applications to Okounkov polytopes of Bott towers and Bott-Samelson varieties.

I am grateful to Dave Anderson, Joel Kamnitzer, Kiumars Kaveh and Askold Khovanskii for useful discussions.

\section{Main CONSTRUCTION}

\subsection{String spaces and parapolytopes.}

Definition 1. A string space of rank $r$ is a real vector space $\mathbb{R}^{d}$ together with a direct sum decomposition

$$
\mathbb{R}^{d}=\mathbb{R}^{d_{1}} \oplus \ldots \oplus \mathbb{R}^{d_{r}}
$$

and a collection of linear functions $l_{1}, \ldots, l_{r} \in\left(\mathbb{R}^{d}\right)^{*}$ such that $l_{i}$ vanishes on $\mathbb{R}^{d_{i}}$.

We choose coordinates in $\mathbb{R}^{d}$ such that they are compatible with the direct sum decomposition. The coordinates will be denoted by $\left(x_{1}^{1}, \ldots, x_{d_{1}}^{1} ; \ldots ; x_{1}^{r}, \ldots, x_{d_{r}}^{r}\right)$ so that the summand $\mathbb{R}^{d_{i}}$ is given by vanishing of all coordinates except for $x_{1}^{i}, \ldots, x_{d_{i}}^{i}$. In what follows, we regard $\mathbb{R}^{d}$ as an affine space. 
Let $\mu=\left(\mu_{1}, \ldots, \mu_{d_{i}}\right)$ and $\nu=\left(\nu_{1}, \ldots, \nu_{d_{i}}\right)$ be two collections of real numbers such that $\mu_{j} \leq \nu_{j}$ for all $j=1, \ldots, d_{i}$. By the coordinate parallelepiped $\Pi(\mu, \nu) \subset \mathbb{R}^{d_{i}}$ we mean the parallelepiped

$$
\Pi(\mu, \nu)=\left\{\left(x_{1}^{i}, \ldots, x_{d_{i}}^{i}\right) \in \mathbb{R}^{d_{i}} \mid \mu_{j} \leq x_{j}^{i} \leq \nu_{j}, j=1, \ldots, d_{i}\right\} .
$$

Definition 2. A convex polytope $P \subset \mathbb{R}^{d}$ is called a parapolytope if for $i=1, \ldots, r$, and a vector $c \in \mathbb{R}^{d}$ the intersection of $P$ with the parallel translate $c+\mathbb{R}^{d_{i}}$ of $\mathbb{R}^{d_{i}}$ is the parallel translate of a coordinate parallelepiped, i.e.,

$$
P \cap\left(c+\mathbb{R}^{d_{i}}\right)=c+\Pi\left(\mu_{c}, \nu_{c}\right)
$$

for $\mu_{c}$ and $\nu_{c}$ that depend on $c$.

For instance, if $d=r$ (i.e., $d_{1}=\ldots=d_{r}=1$ ) then every polytope is a parapolytope. Below is a less trivial example of a parapolytope in a string space.

Example 2.1. Consider the string space

$$
\mathbb{R}^{d}=\mathbb{R}^{n-1} \oplus \mathbb{R}^{n-2} \oplus \ldots \oplus \mathbb{R}^{1}
$$

of rank $r=(n-1)$ and dimension $d=\frac{n(n-1)}{2}$.

Let $\lambda=\left(\lambda_{1}, \ldots, \lambda_{n}\right)$ be a non-increasing collection of integers. For each $\lambda$, define the Gelfand-Zetlin polytope $Q_{\lambda}$ by the inequalities

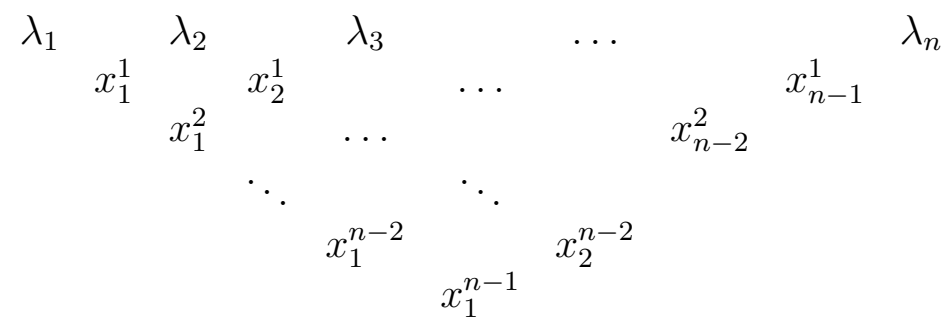

where the notation

$$
c^{b}
$$

means $a \geq c \geq b$. It is easy to check that $Q_{\lambda}$ is a parapolytope. Indeed, consider a parallel translate of $\mathbb{R}^{n-i}$ by the vector $c=\left(c_{1}^{1}, \ldots, c_{n-1}^{1} ; \ldots ; c_{1}^{n-1}\right)$. Put $c_{i}^{0}=\lambda_{i}$ for $i=1, \ldots, n$. The intersection of $Q_{\lambda}$ with $c+\mathbb{R}^{n-i}$ is given by the the following inequalities:

$$
\begin{array}{ccccccccc}
c_{1}^{i-1} & & c_{2}^{i-1} & & c_{3}^{i-1} & & \ldots & & c_{n-i+1}^{i-1} \\
& x_{1}^{i} & & x_{2}^{i} & & \ldots & & x_{n-i}^{i} & \\
& & c_{1}^{i+1} & & \ldots & & c_{n-i-1}^{i+1} & &
\end{array} .
$$

Therefore, the intersection can be identified with the coordinate parallelepiped $c+$ $\Pi(\mu, \nu) \subset c+\mathbb{R}^{n-i}$, where $\mu_{j}=\max \left(c_{j}^{i-1}, c_{j-1}^{i+1}\right)$ and $\nu_{j}=\min \left(c_{j+1}^{i-1}, c_{j}^{i+1}\right)$ (put $c_{0}^{i+1}=$ $-\infty$ and $\left.c_{n-i}^{i+1}=+\infty\right)$. 
2.2. Polytopes and convex chains. Consider the set of all convex polytopes in $\mathbb{R}^{d}$. This set can be endowed with the structure of a commutative semigroup using Minkowski sum

$$
P_{1}+P_{2}=\left\{x_{1}+x_{2} \in \mathbb{R}^{d} \mid x_{1} \in P_{1}, x_{2} \in P_{2}\right\}
$$

It is not hard to check that this semigroup has cancelation property. We can also multiply polytopes by positive real numbers using dilation:

$$
\lambda P=\{\lambda x \mid x \in P\}, \quad \lambda \geq 0 .
$$

Hence, we can embed the semigroup $S$ of convex polytopes into its Grothendieck group $V$, which is a real (infinite-dimensional) vector space. The elements of $V$ are called virtual polytopes.

It is easy to check that the set of parapolytopes in $\mathbb{R}^{d}$ is closed under Minkowski sum and under dilations. Hence, we can define the subspace $V_{\square} \subset V$ of virtual parapolytopes in the string space $\mathbb{R}^{d}$.

Example 2.2. If $\mathbb{R}^{d}$ is a string space of rank 1, i.e. $d_{1}=d$, then parapolytopes are coordinate parallelepipeds $\Pi(\mu, \nu)$. Clearly,

$$
\Pi(\mu, \nu)+\Pi\left(\mu^{\prime}, \nu^{\prime}\right)=\Pi\left(\mu+\mu^{\prime}, \nu+\nu^{\prime}\right) .
$$

Hence, virtual parapolytopes can be identified with the pairs of vectors $\mu, \nu \in \mathbb{R}^{d}$. This yields an isomorphism $V_{\square} \simeq \mathbb{R}^{d} \oplus \mathbb{R}^{d}$. Under this isomorphism, the semigroup of (true) coordinate parallelepipeds gets mapped to the convex cone in $\mathbb{R}^{d} \oplus \mathbb{R}^{d}$ given by the inequalities $\mu_{i} \leq \nu_{i}$ for $i=1, \ldots, d$.

We now define the space $\tilde{V}$ of convex chains following [PKh]. A convex chain is a function on $\mathbb{R}^{d}$ that can be represented as a finite linear combination

$$
\sum_{P} c_{P} \mathbb{I}_{P}
$$

where $c_{P} \in \mathbb{R}$, and $\mathbb{I}_{P}$ is the characteristic function of a convex polytope $P \subset \mathbb{R}^{d}$, that is,

$$
\mathbb{I}_{P}(x)=\left\{\begin{array}{ll}
1, & x \in P \\
0, & x \notin P
\end{array} .\right.
$$

The semigroup $S$ of convex polytopes can be naturally embedded into $\tilde{V}$ :

$$
\iota: S \hookrightarrow \tilde{V} ; \quad \iota: P \mapsto \mathbb{I}_{P}
$$

In what follows, we will work in the space of convex chains and freely identify a polytope $P$ with the corresponding convex chain $\mathbb{I}_{P}$. However, note that the embedding $\iota$ is not a homomorphism, that is, $\mathbb{I}_{P+Q} \neq \mathbb{I}_{P}+\mathbb{I}_{Q}$ (the sum of convex chains is defined as the usual sum of functions). 
Remark 2.3. The embedding $\iota: S \hookrightarrow \tilde{V}$ can be extended to the space $V$ of all virtual polytopes. Namely, there exists a commutative operation $*$ on $\tilde{V}$ (called product of convex chains) such that

$$
\mathbb{I}_{P+Q}=\mathbb{I}_{P} * \mathbb{I}_{Q}
$$

for any two convex polytopes $P$ and $Q$ (see [PKh, Section 2, Proposition-Definition $3]$ ). Virtual polytopes can be identified with the convex chains that are invertible with respect to $*$.

Similarly to the space of convex chains, define the subspace $\tilde{V}_{\square} \subset \tilde{V}$ of convex parachains using only parapolytopes instead of all polytopes. We will use repeatedly the following example of a parachain.

Example 2.4. Consider the simplest case $d=1$. Let $[\mu, \nu] \subset \mathbb{R}$ be a segment (i.e., $\mu<\nu)$, and $[\nu, \mu]$ - a virtual segment. Using the existence of the operation * satisfying $(M)$, it is easy to check that

$$
\iota([\nu, \mu])=-\mathbb{I}_{[-\nu,-\mu]}+\mathbb{I}_{\{-\nu\}}+\mathbb{I}_{\{-\mu\}}
$$

(note that the right hand side is the characteristic function of the open interval $(-\nu,-\mu))$. Indeed,

$$
\begin{gathered}
\mathbb{I}_{[\mu, \nu]} *\left(-\mathbb{I}_{[-\nu,-\mu]}+\mathbb{I}_{\{-\nu\}}+\mathbb{I}_{\{-\mu\}}\right)=-\mathbb{I}_{[\mu, \nu]} * \mathbb{I}_{[-\nu,-\mu]}+\mathbb{I}_{[\nu, \mu]} * \mathbb{I}_{\{-\nu\}}+\mathbb{I}_{[\mu, \nu]} * \mathbb{I}_{\{-\mu\}} \\
=-\mathbb{I}_{[\mu-\nu, \nu-\mu]}+\mathbb{I}_{[\mu-\nu, 0]}+\mathbb{I}_{[0, \nu-\mu]}=\mathbb{I}_{\{0\}} .
\end{gathered}
$$

More generally, if $P \subset \mathbb{R}^{d}$ is a convex polytope then

$$
(-1)^{\operatorname{dim} P} \mathbb{I}_{P} * \mathbb{I}_{\text {int }\left(P^{\vee}\right)}=\mathbb{I}_{\{0\}},
$$

where $P^{\vee}=\{-x \mid x \in P\}$, and $\operatorname{int}\left(P^{\vee}\right)$ denotes the interior of $P^{\vee}$ (see [PKh, Section 2, Theorem 2]).

2.3. Divided difference operators on parachains. For each $i=1, \ldots, n$, we now define a divided difference (or Demazure) operator $D_{i}$ on the space of convex parachains $\tilde{V}_{\square}$. It is enough to define $D_{i}$ on convex parapolytopes and then extend the definition by linearity to the other parachains. Let $P$ be a parapolytope. Choose the smallest $j=1, \ldots, d_{i}$ such that $P$ lies in the hyperplane $\left\{x_{j}^{i}=\right.$ const $\}$. If no such $j$ exists, then $D_{i}\left(\mathbb{I}_{P}\right)$ is not defined. Otherwise, we expand $P$ in the direction of $x_{j}^{i}$ as follows.

First, suppose that a parapolytope $P$ lies in $\left(c+\mathbb{R}^{d_{i}}\right)$ for some $c \in \mathbb{R}^{d}$, i.e., $P=$ $c+\Pi(\mu, \nu)$ is a coordinate parallelepiped. We always fix the choice of $c$ by requiring that $c$ lies in the direct complement to $\mathbb{R}^{d_{i}}$ with respect to the decomposition $\mathbb{R}^{d}=$ $\mathbb{R}^{d_{1}} \oplus \ldots \oplus \mathbb{R}^{d_{i}} \oplus \ldots \oplus \mathbb{R}^{d_{r}}$. Consider $\nu^{\prime}=\left(\nu_{1}^{\prime}, \ldots, \nu_{d_{i}}^{\prime}\right)$, where $\nu_{k}^{\prime}=\nu_{k}$ for all $k \neq j$, and $\nu_{j}^{\prime}$ is defined by the equality

$$
\sum_{k=1}^{d_{i}}\left(\mu_{k}+\nu_{k}^{\prime}\right)=l_{i}(c) .
$$


If $\nu_{j}^{\prime} \geq \nu_{j}$, then $D_{i}^{+}(P):=c+\Pi\left(\mu, \nu^{\prime}\right)$ is a true coordinate parallelepiped. Note that $P$ is a facet of $D_{i}^{+}(P)$ unless $\nu^{\prime}=\nu$

If $\nu_{j}^{\prime}<\nu_{j}$, define $\mu^{\prime}=\left(\mu_{1}^{\prime}, \ldots, \mu_{d_{i}}^{\prime}\right)$ by setting $\mu_{k}^{\prime}=\mu_{k}$ for all $k \neq j$, and $\mu_{j}^{\prime}=\nu_{j}^{\prime}$. Then $D_{i}^{-}(P):=c+\Pi\left(\mu^{\prime}, \nu\right)$ is a true coordinate parallelepiped, and $P$ is a facet of $D_{i}^{-}(P)$. Let $P^{\prime}$ be the facet of $D_{i}^{-}(P)$ parallel to $P$.

We now define $D_{i}\left(\mathbb{I}_{P}\right)$ as follows:

$$
D_{i}\left(\mathbb{I}_{P}\right)= \begin{cases}\mathbb{I}_{D_{i}^{+}(P)} & \text { if } \nu_{j} \leq \nu_{j}^{\prime}, \\ -\mathbb{I}_{D_{i}^{-}(P)}+\mathbb{I}_{P}+\mathbb{I}_{P^{\prime}} & \text { if } \nu_{j}>\nu_{j}^{\prime}\end{cases}
$$

Remark 2.5. This definition is motivated by the following observation. Let $\mu$ and $\nu$ be integers such that $\mu<\nu$. Define the function $f(\mu, \nu, t)$ of a complex variable $t$ by the formula

$$
f(\mu, \nu, t)=t^{\mu}+t^{\mu+1}+\ldots+t^{\nu},
$$

that is, $f$ is the exponential sum over all integer points in the segment $[\mu, \nu] \subset \mathbb{R}$. Computing the sum of the geometric progression, we get that

$$
f(\mu, \nu, t)=\frac{t^{\mu}-t^{\nu+1}}{1-t}
$$

This formula gives a meromorphic continuation of $f(\mu, \nu, t)$ to all real $\mu$ and $\nu$. In particular, for integer $\mu$ and $\nu$ such that $\mu>\nu$ we obtain

$$
f(\mu, \nu, t)=\frac{t^{\mu}-t^{\nu+1}}{1-t}=-\left(t^{\nu+1}+\ldots+t^{\mu-1}\right),
$$

that is, $f$ is minus the exponential sum over all integer points in the open interval $(\nu, \mu) \subset \mathbb{R}$ (cf. Example 2.4).

Definition 3 . For an arbitrary parapolytope $P \subset \mathbb{R}^{d}$ define $D_{i}\left(\mathbb{I}_{P}\right)$ by setting

$$
\left.D_{i}\left(\mathbb{I}_{P}\right)\right|_{c+\mathbb{R}^{d_{i}}}=D_{i}\left(\mathbb{I}_{P \cap\left(c+\mathbb{R}^{d_{i}}\right)}\right)
$$

for all $c$ in the complement to $\mathbb{R}^{d_{i}}$.

It is not hard to check that this definition yields a convex chain. In many cases (see examples below and in Section 3 ),$D_{i}\left(\mathbb{I}_{P}\right)$ is the characteristic function of a polytope (and $P$ is a facet of this polytope unless $\left.D_{i}\left(\mathbb{I}_{P}\right)=\mathbb{I}_{P}\right)$. This polytope will be denoted by $D_{i}(P)$.

The definition immediately implies that similarly to the classical Demazure operators the convex-geometric ones satisfy the identity $D_{i}^{2}=D_{i}$. It would be interesting to find an analog of braid relations for these operators.

\subsection{Examples.}



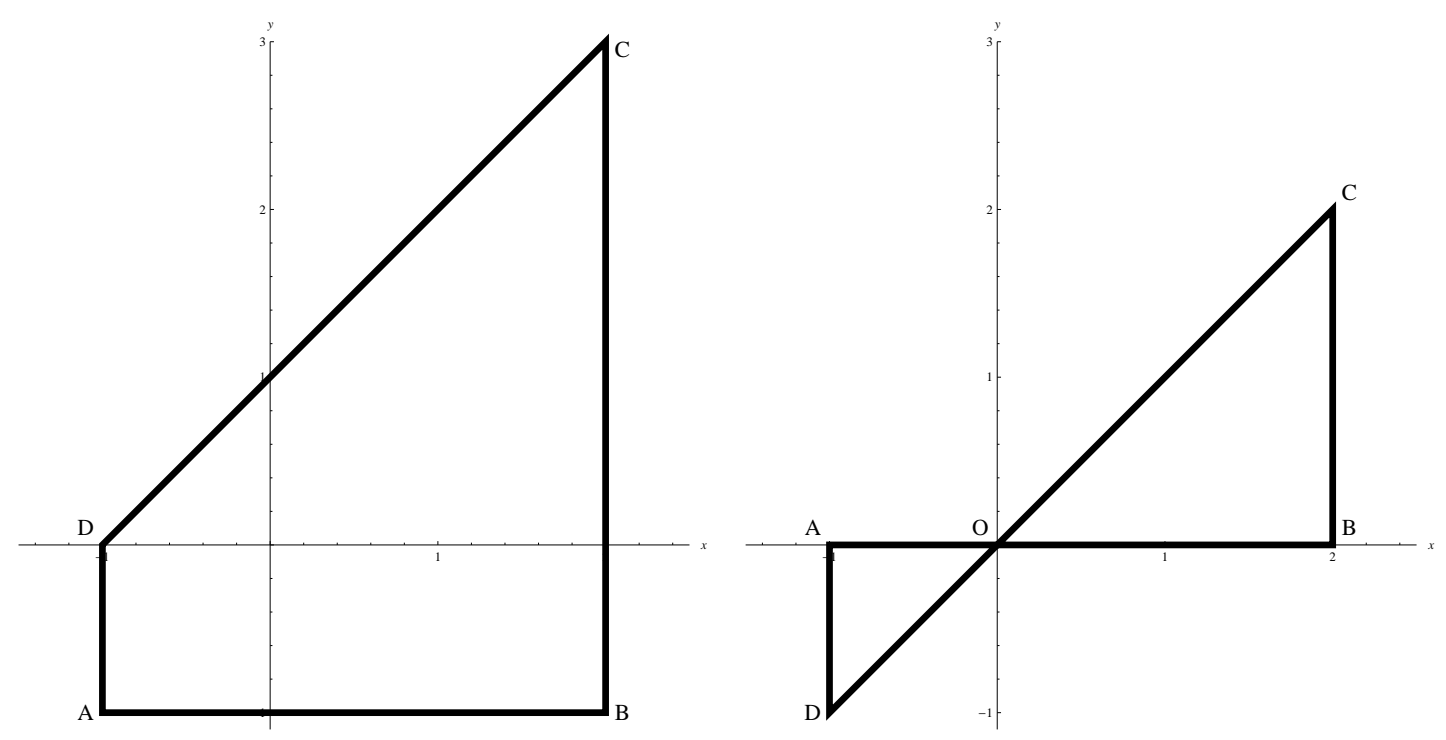

FiguRE 1. Trapezoids $D_{2}(P)$ for different segments $P=A B$.

Dimension 2. The simplest meaningful example is $\mathbb{R}^{2}=\mathbb{R} \oplus \mathbb{R}$. Label coordinates in $\mathbb{R}^{2}$ by $x:=x_{1}^{1}$ and $y:=x_{2}^{1}$. Assume that $l_{1}=y$ and $l_{2}=x$. If $P=\left\{\left(\mu_{1}, \mu_{2}\right)\right\}$ is a point, and $\mu_{2} \geq 2 \mu_{1}$, then $D_{1}(P)$ is a segment:

$$
D_{1}(P)=\left[\left(\mu_{1}, \mu_{2}\right),\left(\mu_{2}-\mu_{1}, \mu_{2}\right)\right]
$$

If $\mu_{2}<2 \mu_{1}$, then $D_{1}\left(\mathbb{I}_{P}\right)$ is a virtual segment, that is,

$$
D_{1}\left(\mathbb{I}_{P}\right)=-\mathbb{I}_{\left[\left(\mu_{2}-\mu_{1}, \mu_{2}\right),\left(\mu_{1}, \mu_{2}\right)\right]}+\mathbb{I}_{P}+\mathbb{I}_{\left(\mu_{2}-\mu_{1}, \mu_{2}\right)} .
$$

If $P=A B$ is a horizontal segment, where $A=\left(\mu_{1}, \mu_{2}\right)$ and $B=\left(\nu_{1}, \mu_{2}\right)$, then $D_{2}(P)$ is the trapezoid $A B C D$ given by the inequalities

$$
\mu_{1} \leq x \leq \nu_{1}, \quad \mu_{2} \leq y \leq x-\mu_{2} .
$$

See Figure 1 for $D_{2}(P)$ in the case $\mu_{1}=-1, \nu_{1}=2, \mu_{2}=-1$ (left) and $\mu_{1}=-1$, $\nu_{1}=2, \mu_{2}=0$ (right). In the latter case, the convex chain $D_{2}\left(\mathbb{I}_{P}\right)$ is equal to

$$
\mathbb{I}_{O B C}-\mathbb{I}_{A D O}+\mathbb{I}_{O A}+\mathbb{I}_{D O}-\mathbb{I}_{O} \text {. }
$$

Dimension 3. A more interesting example is $\mathbb{R}^{3}=\mathbb{R}^{2} \oplus \mathbb{R}$. Label coordinates in $\mathbb{R}^{3}$ by $x:=x_{1}^{1}, y:=x_{2}^{1}$ and $z:=x_{1}^{2}$. Assume that $l_{1}=z$ and $l_{2}=x+y$. If $P=\left(\mu_{1}, \mu_{2}, \mu_{3}\right)$ is a point, then $D_{1}(P)$ is a segment:

$$
D_{1}(P)=\left[\left(\mu_{1}, \mu_{2}, \mu_{3}\right),\left(\mu_{3}-\mu_{1}-2 \mu_{2}, \mu_{2}, \mu_{3}\right)\right] .
$$

Similarly, if $P=\left[\left(\mu_{1}, \mu_{2}, \mu_{3}\right),\left(\nu_{1}, \mu_{2}, \mu_{3}\right)\right]$ is a segment in $\mathbb{R}^{2}$, then $D_{1}(P)$ is the rectangle given by the equation $z=\mu_{3}$ and the inequalities

$$
\mu_{1} \leq x \leq \nu_{1}, \quad \mu_{2} \leq y \leq \mu_{3}-\mu_{1}-\nu_{1}-\mu_{2} .
$$



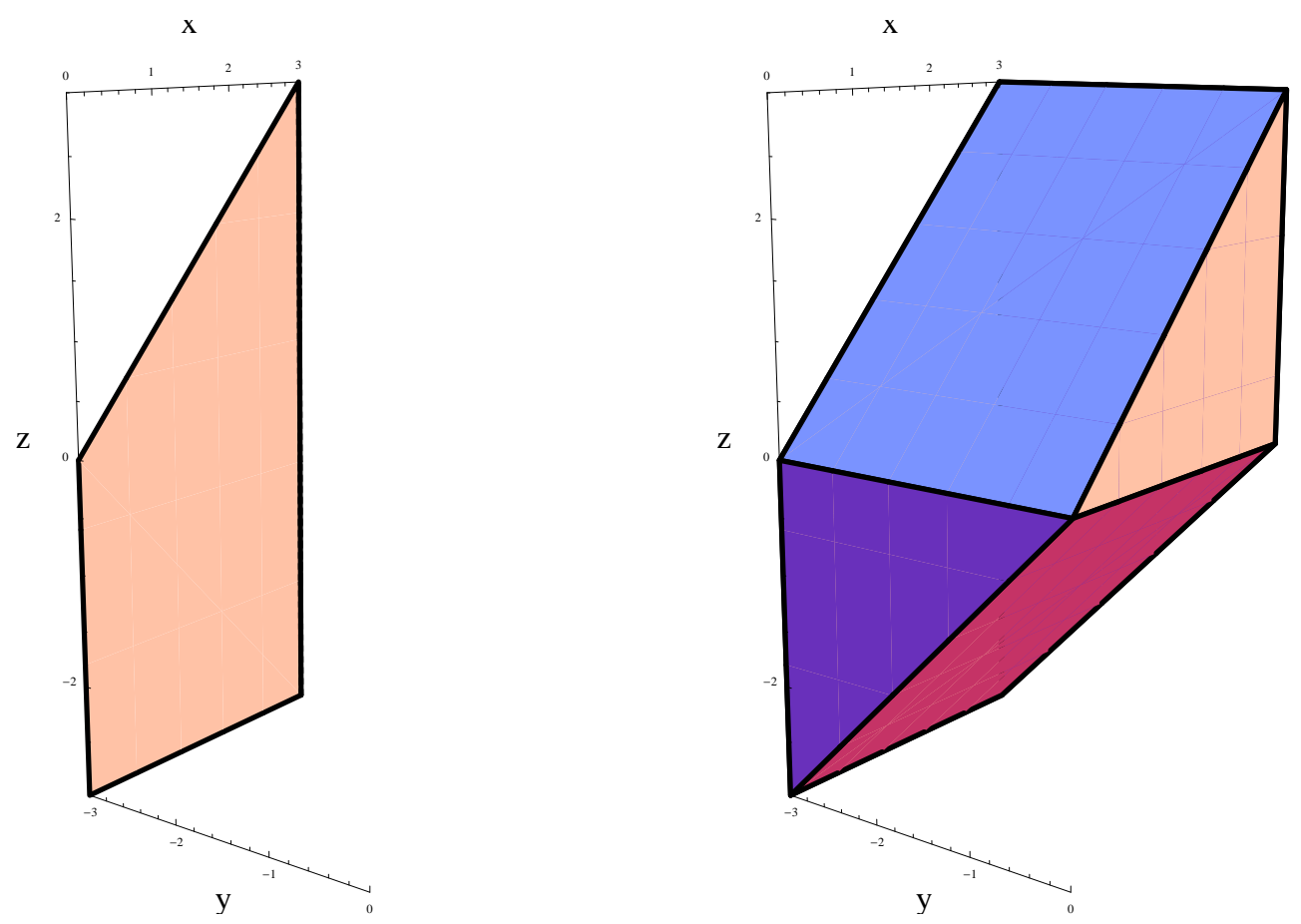

Figure 2. Trapezoid $D_{2} D_{1}(P)$ and polytope $D_{1} D_{2} D_{1}(P)$ for a point $P=(0,-3,-3)$

Using the previous calculations, it is easy to show that if $P=\left(\lambda_{2}, \lambda_{3}, \lambda_{3}\right)$ is a point and $\lambda_{3}<\lambda_{2}<-\lambda_{2}-\lambda_{3}$, then $D_{1} D_{2} D_{1}(P)$ is the 3-dimensional Gelfand-Zetlin polytope $Q_{\lambda}$ (as defined in Example 2.1) for $\lambda=\left(\lambda_{1}, \lambda_{2}, \lambda_{3}\right)$, where $\lambda_{1}=-\lambda_{2}-\lambda_{3}$. Indeed, $D_{2} D_{1}(P)$ is the trapezoid (see Figure 2) given by the equation $y=\lambda_{3}$ and the inequalities

$$
\lambda_{2} \leq x \leq \lambda_{1}, \quad \lambda_{3} \leq z \leq x .
$$

Then $D_{1} D_{2} D_{1}(P)$ is the union of all rectangles $D_{2}\left(I_{a}\right)$ for $a \in\left[\lambda_{3}, \lambda_{1}\right]$, where $I_{a}$ is the segment $D_{2} D_{1}(P) \cap\{z=a\}$, that is, $I_{a}=\left[\left(\max \left\{z, \lambda_{2}\right\}, \lambda_{3}, a\right),\left(\lambda_{1}, \lambda_{2}, a\right)\right]$. Hence,

$$
\lambda_{3} \leq y \leq \min \left\{\lambda_{2}, z\right\}
$$

Similarly to the last example, we construct Gelfand-Zetlin polytopes for arbitrary $n$ using the string space from Example 2.1 (see Theorem 3.4).

\section{Polytopes and Demazure characters}

3.1. Characters of polytopes. For a string space $\mathbb{R}^{d}=\mathbb{R}^{d_{1}} \oplus \ldots \oplus \mathbb{R}^{d_{r}}$, denote by $\sigma_{i}(x)$ the sum of the coordinates of $x \in \mathbb{R}^{d}$ that correspond to the subspace $\mathbb{R}^{d_{i}}$, i.e., $\sigma_{i}(x)=\sum_{k=1}^{d_{i}} x_{k}^{i}$. With each integer point $x \in \mathbb{R}^{d}$ in the string space, we associate the weight $p(x) \in \mathbb{R}^{r}$ defined as $\left(\sigma_{1}(x), \ldots, \sigma_{r}(x)\right)$. For the rest of the paper, we will always assume that $l_{i}(x)$ depends only on $p(x)$, that is, $l_{i}$ comes from a linear 
function on $\mathbb{R}^{r}$ (the latter will also be denoted by $l_{i}$ ). In addition, we assume that $l_{i}$ is integral, i.e., $l_{i}(x) \in \mathbb{Z}$ for all $x \in \mathbb{Z}^{n}$.

Denote the basis vectors in $\mathbb{R}^{r}$ by $\alpha_{1}, \ldots, \alpha_{r}$, and denote the coordinates with respect to this basis by $\left(y_{1}, \ldots, y_{r}\right)$. For each $i=1, \ldots r$, define the affine reflection $s_{i}: \mathbb{R}^{r} \rightarrow \mathbb{R}^{r}$ by the formula

$$
s_{i}\left(y_{1}, \ldots, y_{i}, \ldots, y_{r}\right)=\left(y_{1}, \ldots, l_{i}(y)-y_{i}, \ldots, y_{r}\right) .
$$

Example 3.1. For the string space $\mathbb{R}^{d}=\mathbb{R}^{n-1} \oplus \mathbb{R}^{n-2} \oplus \ldots \oplus \mathbb{R}^{1}$ from Example 2.1, define the functions $l_{i}$ by the formula

$$
l_{i}(x)=\sigma_{i-1}(x)+\sigma_{i+1}(x),
$$

where we put $\sigma_{0}=\sigma_{n}=0$. Identify $\mathbb{R}^{n-1}$ with the weight lattice of $S L_{n}$ so that $\alpha_{i}$ is identified with the $i$-th simple root. In this case, the reflection $s_{i}$ coincides with the simple reflection in the hyperplane perpendicular to the root $\alpha_{i}$.

We now consider the ring $R$ of Laurent polynomials in the formal exponentials $t_{1}:=e^{\alpha_{1}}, \ldots, t_{n}:=e^{\alpha_{n}}$ (that is, $R$ is the group algebra of the lattice $\mathbb{Z}^{n} \subset \mathbb{R}^{n}$ ). Let $P \subset \mathbb{R}^{d}$ be a lattice polytope in the string space, i.e., the vertices of $P$ belong to $\mathbb{Z}^{n}$. Define the character of $P$ as the sum of formal exponentials $e^{p(x)}$ over all integer points $x$ inside and at the boundary of $P$ :

$$
\chi(P):=\sum_{x \in P \cap \mathbb{Z}^{d}} e^{p(x)}
$$

In particular, if $d=r$, then $\chi(P)$ is exactly the integer point transform of $P$. The $R$-valued function $\chi$ can be extended by linearity to all lattice convex chains, that is, to the chains $\sum_{P} c_{P} \mathbb{I}_{P}$ such that $P$ is a lattice polytope and $c_{P} \in \mathbb{Z}$.

Define Demazure operator $T_{i}$ on $R$ as follows:

$$
\left[T_{i} f\right](y)=\frac{f(y)-t_{i} s_{i} f(y)}{1-t_{i}}
$$

where $s_{i} f(y):=f\left(s_{i} y\right)$. For the string space of Example 3.1, these operators reduce to the classical Demazure operators on the group algebra of the weight lattice of $S L_{n}$.

The following result motivates Definition 3 ,

Theorem 3.2. Let $P \subset \mathbb{R}^{d}$ be a lattice parapolytope. Then

$$
\chi\left(D_{i}\left(\mathbb{I}_{P}\right)\right)=T_{i} \chi(P) .
$$

Proof. By definition of $D_{i}\left(\mathbb{I}_{P}\right)$, it suffices to prove this identity when $P=c+\Gamma$, where $c$ lies in the complement to $\mathbb{R}^{d_{i}}$ and $\Gamma:=\Pi(\mu, \nu) \subset \mathbb{R}^{d_{i}}$ is a coordinate parallelepiped. Then

$$
\chi(P)=e^{p(c)} \sum_{z \in \Gamma \cap \mathbb{Z}^{d_{i}}} t_{i}^{\sigma(z)} .
$$


Hence,

Recall that by definition of $\nu^{\prime}$ we have

$$
T_{i}(\chi(P))=e^{p(c)} T_{i}\left(\sum_{z \in \Gamma \cap \mathbb{Z}^{d_{i}}} t_{i}^{\sigma(z)}\right) .
$$

$$
\sum_{k=1}^{d_{i}}\left(\mu_{k}+\nu_{k}^{\prime}\right)=l_{i}(c)
$$

Assume that $\nu_{j}^{\prime} \geq \nu_{j}$. Let $\Pi$ denote $\Pi\left(\mu, \nu^{\prime}\right)$. Then $\Gamma, \Pi$ and $T_{i}$ satisfy the hypothesis of [KST, Proposition 6.3]. Applying this proposition we get that

$$
T_{i}\left(\sum_{z \in \Gamma \cap \mathbb{Z}^{d_{i}}} t_{i}^{\sigma(z)}\right)=\sum_{z \in \Pi \cap \mathbb{Z}^{d_{i}}} t_{i}^{\sigma(z)} .
$$

Hence, $T_{i}(\chi(P))=\chi\left(D_{i}(P)\right)$.

The case $\nu_{j}^{\prime}<\nu_{j}$ is completely analogous.

Note that Theorem 3.2 for $d_{i}=1$ follows directly from the definitions of $T_{i}$ and $D_{i}$ (see Remark 2.5).

Example 3.3. Figure 3 illustrates Theorem 3.2 when $d_{i}=2$ and $P=c+\Gamma$ where $\Gamma \subset$ $\mathbb{R}^{d_{i}}$ is the segment $[(-1,-1),(2,-1)]$. Namely, $T_{i}\left(t_{i}^{x_{1}^{i}+x_{2}^{i}}\right)$ is equal to the character of the segment $\left[\left(x_{1}^{i}, x_{2}^{i}\right),\left(x_{1}^{i}, l_{i}(c)-2 x_{1}^{i}-x_{2}^{i}\right)\right]$ for every $\left(x_{1}^{i}, x_{2}^{i}\right) \in \Gamma \cap \mathbb{Z}^{2}$ by definition of $T_{i}$. Hence,

$$
\sum_{\left(x_{1}^{i}, x_{2}^{i}\right) \in \Gamma \cap \mathbb{Z}^{2}} T_{i}\left(t_{i}^{x_{1}^{i}+x_{2}^{i}}\right)
$$

for $l_{i}(c)=3$ coincides with the character of the trapezoid shown on Figure 3 (left). It is easy to construct a bijective correspondence between the integer points in the trapezoid and those in the rectangle $D_{i}(P)$ in such a way that the sum of coordinates is preserved. The former are marked by black dots, and the latter by empty circles.

Theorem 3.2 allows one to construct various polytopes (possibly virtual) and convex chains whose characters yield the Demazure characters (in particular, the Weyl character) of irreducible representations of reductive groups (see Section 3.3). The same character can be captured using string spaces for different partitions $d=d_{1}+d_{2}+\ldots+d_{n}$ (see Section 4.3). The case $d_{1}=\ldots=d_{n}=1$ produces polytopes with very simple combinatorics, namely, multidimensional versions of trapezoids that are combinatorially equivalent to cubes (they are called twisted cubes in [GK]). However, twisted cubes that represent the Weyl characters are virtual. Considering string spaces with $d_{i}>1$ allows one to represent the Weyl character by a true though more intricate polytope (see Example 3.4 for $S L_{3}$ and Section 4.3 ). The reason is illustrated by Figure 3 (right) that depicts a virtual trapezoid and a (true) rectangle 

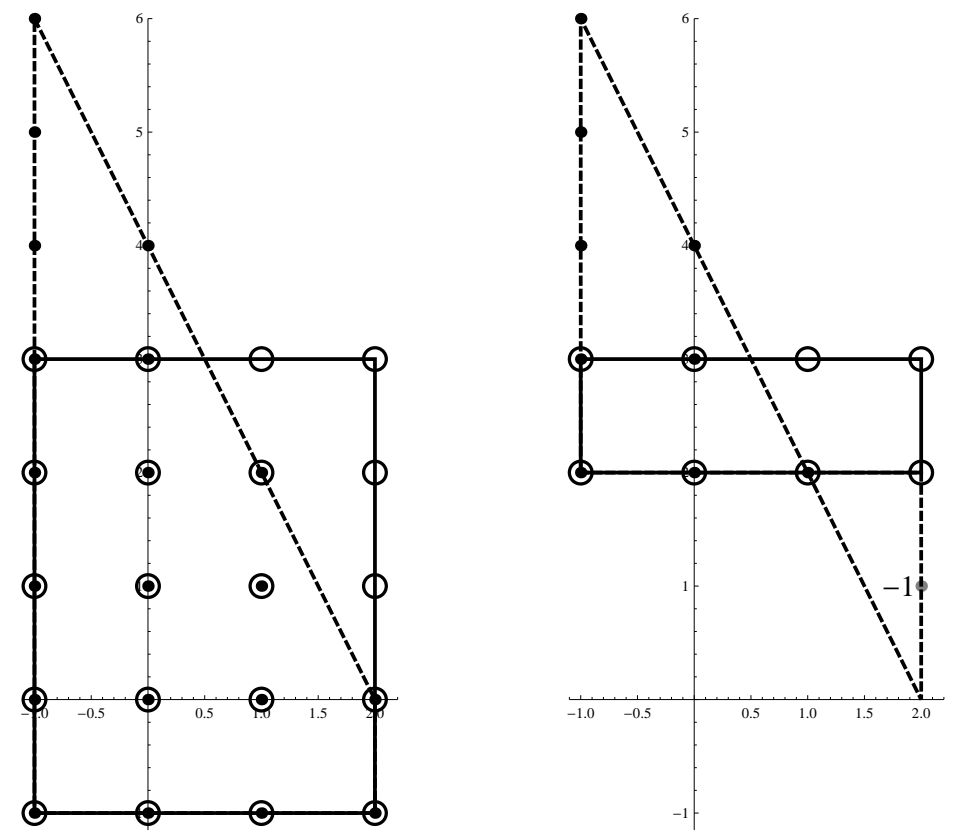

FiguRE 3. Rectangle and trapezoid yield the same character

with the same character. Note that the point $(2,1)$ (marked by -1 ) contributes a negative summand to the character of the trapezoid.

3.2. Gelfand-Zetlin polytopes for $S L_{n}$. Let $\mathbb{R}^{d}=\mathbb{R}^{n-1} \oplus \mathbb{R}^{n-2} \oplus \ldots \oplus \mathbb{R}^{1}$ be the string space of rank $(n-1)$ from Example 3.1. The theorem below shows how to construct the classical Gelfand-Zetlin polytopes (see Example 2.1) via the convexgeometric Demazure operators $D_{1}, \ldots, D_{n-1}$.

Theorem 3.4. For every strictly dominant weight $\lambda=\left(\lambda_{1}, \ldots, \lambda_{n}\right)$ (that is, $\lambda_{1}>$ $\ldots>\lambda_{n}$ ) of $G L_{n}$ such that $\lambda_{1}+\ldots+\lambda_{n}=0$, the Gelfand-Zetlin polytope $Q_{\lambda}$ coincides with the polytope

$$
\left[\left(D_{1}\right)\left(D_{2} D_{1}\right)\left(D_{3} D_{2} D_{1}\right) \ldots\left(D_{n-1} \ldots D_{1}\right)\right]\left(a_{\lambda}\right)
$$

where $a_{\lambda} \in \mathbb{R}^{d}$ is the point $\left(\lambda_{2}, \ldots, \lambda_{n} ; \lambda_{3}, \ldots, \lambda_{n} ; \ldots ; \lambda_{n}\right)$.

Proof. Let us define the polytope

$$
P_{\lambda}(i, j):=\left[\left(\hat{D}_{n-j} \ldots \hat{D}_{i} D_{i-1} \ldots D_{1}\right) \ldots\left(D_{n-1} \ldots D_{1}\right)\right]\left(a_{\lambda}\right)
$$

for every pair $(i, j)$ such that $1 \leq i \leq(n-j) \leq(n-1)$. Put $x_{l}^{0}=\lambda_{i}$ for $l=1, \ldots$, $n$. We will show by induction on dimension that $P_{\lambda}(i, j)$ is the face of the GelfandZetlin polytope $Q_{\lambda}$ given by the equations $x_{l}^{k}=x_{l+1}^{k-1}$ for all pairs $(k, l)$ such that either $l>j$, or $l=j$ and $k \geq i$. The induction base is $P_{\lambda}(1,1)=a_{\lambda}$, which is clearly a vertex of $Q_{\lambda}$ by our assumption. The induction step follows from Lemma 
3.5 below. Hence, $P_{\lambda}(1, n-1)$ is the facet of $Q_{\lambda}$ given by the equation $x_{n-1}^{1}=\lambda_{n}$. Applying Lemma 3.5 again, we get that $D_{1}\left(P_{\lambda}(1, n-1)\right)=Q_{\lambda}$.

Note that any Gelfand-Zetlin polytope $Q_{\lambda}$ can be obtained by a parallel translation from one with $\lambda_{1}+\ldots+\lambda_{n}=0$.

The lemma below can be easily deduced directly from the definition of $D_{i}$ using Example 2.1 together with an evident observation that $a+b=\min \{a, b\}+\max \{a, b\}$ for any $a, b \in \mathbb{R}$.

Lemma 3.5. Let $\Gamma$ be a face of the Gelfand-Zetlin polytope $Q_{\lambda}$ given by the following equations

$$
\begin{aligned}
& \begin{array}{lllllllll}
x_{1}^{i-1} & x_{2}^{i-1} & x_{3}^{i-1} & \ldots & x_{j}^{i-1} & x_{j+1}^{i-1} & x_{j+2}^{i-1} & \ldots & x_{n-i+1}^{i-1} \\
& & & & &
\end{array} \\
& \begin{array}{lllllllll}
x_{1}^{i} & x_{2}^{i} & \ldots & x_{j-1}^{i} & x_{j}^{i} & x_{j+1}^{i} & \ldots & x_{n-i}^{i} \quad .
\end{array} \\
& \begin{array}{lllllll}
x_{1}^{i+1} & \ldots & x_{j-2}^{i+1} & x_{j-1}^{i+1} & x_{j}^{i+1} & \ldots & x_{n-i-1}^{i+1}
\end{array}
\end{aligned}
$$

as well as by (possibly) other equations that do not involve variables $x_{1}^{i}, \ldots x_{n-i}^{i}$. Then the defining equations of $D_{i}(\Gamma)$ are obtained from those of $\Gamma$ by removing the equation $x_{j}^{i}=x_{j+1}^{i-1}$.

Recall that integer points inside and at the boundary of the Gelfand-Zetlin polytope $Q_{\lambda}$ by definition of this polytope parameterize a natural basis (Gelfand-Zetlin basis) in the irreducible representation of $G L_{n}$ with the highest weight $\lambda$. Under this correspondence, the map $p: \mathbb{R}^{d} \rightarrow \mathbb{R}^{n-1}$ assigns to every integer point the weight of the corresponding basis vector. Combining Theorem 3.4 with Theorem 3.2 one gets a combinatorial proof of the Demazure character formula for the decomposition $w_{0}=\left(s_{1}\right)\left(s_{2} s_{1}\right)\left(s_{3} s_{2} s_{1}\right) \ldots\left(s_{n-1} s_{n-2} \ldots s_{1}\right)$ of the longest word in $S_{n}$ (in this case, the Demazure character coincides with the Weyl character of $V_{\lambda}$ ). Here $s_{i}$ denotes the elementary transposition $(i, i+1) \in S_{n}$.

3.3. Applications to arbitrary reductive groups. We now generalize GelfandZetlin polytopes to other reductive groups using Theorem 3.2 . Let $G$ be a connected reductive group of semisimple rank $r$. Let $\alpha_{1}, \ldots, \alpha_{r}$ denote simple roots of $G$, and $s_{1}, \ldots, s_{r}$ the corresponding simple reflections. Fix a reduced decomposition $w_{0}=s_{i_{1}} s_{i_{2}} \cdots s_{i_{d}}$ where $w_{0}$ is the longest element of the Weyl group of $G$. Let $d_{i}$ be the number of $s_{i_{j}}$ in this decomposition such that $i_{j}=i$. Consider the string space

$$
\mathbb{R}^{d}=\mathbb{R}^{d_{1}} \oplus \ldots \oplus \mathbb{R}^{d_{r}}
$$

where the functions $l_{i}$ are given by the formula:

$$
l_{i}(x)=\sum_{k \neq i}\left(\alpha_{k}, \alpha_{i}\right) \sigma_{k}(x) .
$$

Here $\left(\alpha_{k}, \alpha_{i}\right)$ is determined by the simple reflection $s_{i}$ as follows:

$$
s_{i}\left(\alpha_{k}\right)=\alpha_{k}+\left(\alpha_{k}, \alpha_{i}\right) \alpha_{i}
$$


(that is, the function $\left(\cdot, \alpha_{i}\right)$ is minus the coroot corresponding to $\alpha_{i}$ ). In particular, if $G=S L_{n}$ and $w_{0}=\left(s_{1}\right)\left(s_{2} s_{1}\right)\left(s_{3} s_{2} s_{1}\right) \ldots\left(s_{n-1} \ldots s_{1}\right)$, then we get the string space from Example 3.1.

Define the projection $p$ of the string space to the real span $\mathbb{R}^{r}$ of the weight lattice of $G$ by the formula $p(x)=\sigma_{1}(x) \alpha_{1}+\ldots+\sigma_{r}(x) \alpha_{r}$.

Theorem 3.6. For every dominant weight $\lambda$ in the root lattice of $G$, and every point $a_{\lambda} \in \mathbb{Z}^{d}$ such that $p\left(a_{\lambda}\right)=w_{0} \lambda$ the convex chain

$$
P_{\lambda}:=D_{i_{1}} D_{i_{2}} \ldots D_{i_{d}}\left(a_{\lambda}\right)
$$

yields the Weyl character $\chi\left(V_{\lambda}\right)$ of the irreducible $G$-module $V_{\lambda}$, that is,

$$
\chi\left(V_{\lambda}\right)=\chi\left(P_{\lambda}\right) .
$$

Proof. By the Demazure character formula Andersen we have

$$
\chi\left(V_{\lambda}\right)=T_{i_{1}} \ldots T_{i_{d}} e^{w_{0} \lambda} .
$$

This formula together with Theorem 3.2 implies by induction the desired statement.

As a corollary, we get that $p_{*}\left(P_{\lambda}\right)$ is the weight polytope of $V_{\lambda}$ in $\mathbb{R}^{r}$. Here $p_{*}$ denotes the push-forward of convex chains (see [PKh, Proposition-Definition 2]).

Remark 3.7. A slight modification of Theorem 3.2 makes it applicable to all dominant weights (not only those inside the root lattice). Namely, instead of the lattices $\mathbb{Z}^{d} \subset \mathbb{R}^{d}$ and $\mathbb{Z}^{r} \subset \mathbb{R}^{r}$ one should consider the shifted lattices $a_{\lambda}+\mathbb{Z}^{d} \subset \mathbb{R}^{d}$ and $\lambda+\mathbb{Z}^{r} \subset \mathbb{R}^{r}$, and define characters of polytopes with respect to these new lattices. The convex chain $P_{\lambda}$ will be lattice with respect to the lattice $a_{\lambda}+\mathbb{Z}^{d}$.

In the same way, we can construct convex chains that capture the characters of Demazure submodules of $V_{\lambda}$ for any element $w$ in the Weyl group and a reduced decomposition $w=s_{j_{1}} \ldots s_{j_{\ell}}$ (see Corollary 4.5). In particular, if $s_{j_{1}} \ldots s_{j_{\ell}}$ is a terminal subword of $s_{i_{1}} s_{i_{2}} \cdots s_{i_{d}}$ (that is, $j_{\ell}=i_{d}, j_{\ell-1}=i_{d-1}$, etc.) then the corresponding convex chain is a face of $P_{\lambda}$. It is interesting to check whether this convex chain is always a true polytope. One way to do this would be to identify it with an Okounkov polytope of the Bott-Samelson resolution corresponding to the word $s_{j_{1}} \ldots s_{j_{\ell}}$ (see Conjecture 4.1).

\subsection{Examples.}

$\operatorname{Sp}(4)$. Take $G=S p(4)$ and $w_{0}=s_{2} s_{1} s_{2} s_{1}$ (here $\alpha_{1}$ denotes the shorter root and $\alpha_{2}$ denotes the longer one). The corresponding string space of rank 2 is $\mathbb{R}^{4}=\mathbb{R}^{2} \oplus \mathbb{R}^{2}$ together with $l_{1}=2\left(x_{1}^{2}+x_{2}^{2}\right)$ and $l_{2}=x_{1}^{1}+x_{2}^{1}$. Let $\lambda=-p_{1} \alpha_{1}-p_{2} \alpha_{2}$ be a dominant weight, that is, $\lambda_{1}:=\left(p_{2}-p_{1}\right) \geq 0$ and $\lambda_{2}:=\left(p_{1}-2 p_{2}\right) \geq 0$. Choose a point $a_{\lambda}=(a, b, c, d)$ such that $(a+b)=p_{1}$ and $(c+d)=p_{2}$ (that is, $\left.p\left(a_{\lambda}\right)=w_{0} \lambda=-\lambda\right)$. Label coordinates in $\mathbb{R}^{4}$ by $x:=x_{1}^{1}, y:=x_{2}^{1}, z:=x_{1}^{2}$ and $t:=x_{2}^{2}$. Then the polytope $D_{2} D_{1} D_{2} D_{1}\left(a_{\lambda}\right)$ is given by inequalities

$$
0 \leq x-a \leq 2 \lambda_{1}, \quad z-c \leq x-a+\lambda_{2}, \quad y-b \leq 2(z-c),
$$




$$
y-b \leq z-c+\lambda_{2}, \quad 0 \leq t-d \leq \lambda_{2}, \quad t-d \leq \frac{y-b}{2} .
$$

It is not hard to show that the polytopes $D_{1} D_{2} D_{1} D_{2}\left(a_{\lambda}\right)$ and $D_{2} D_{1} D_{2} D_{1}\left(a_{\lambda}\right)$ are the same up to a linear transformation of $\mathbb{R}^{4}$. Each polytope has 11 vertices, hence, they are not combinatorially equivalent to string polytopes for $s_{1} s_{2} s_{1} s_{2}$ or $s_{2} s_{1} s_{2} s_{1}$ defined in [L].

$\mathbf{S L}(3)$. Take $G=S L(3)$ and $w_{0}=s_{1} s_{2} s_{1}$. The corresponding string space of rank 2 coincides with the one from Section 2.4 , namely, $\mathbb{R}^{3}=\mathbb{R}^{2} \oplus \mathbb{R}$, and $l_{1}=x_{1}^{2}$, $l_{2}=x_{1}^{1}+x_{2}^{1}$. If $a_{\lambda}=(b, c, c)$ where $-b-c \geq b \geq c$, then the polytope $D_{1} D_{2} D_{1}\left(a_{\lambda}\right)$ is the Gelfand-Zetlin polytope $Q_{\lambda}$ for $\lambda=(-b-c, b, c)$. Label coordinates in $\mathbb{R}^{3}$ by $x:=x_{1}^{1}, y:=x_{2}^{1}$ and $z:=x_{1}^{2}$. We now introduce a different structure of a string space in $\mathbb{R}^{3}$ by splitting $\mathbb{R}^{2}$, namely, $\mathbb{R}^{3}=\mathbb{R}^{1} \oplus \mathbb{R}^{1} \oplus \mathbb{R}^{1}$ with coordinates $\tilde{x}_{1}^{1}, \tilde{x}_{1}^{2}, \tilde{x}_{1}^{3}$ such that $\tilde{x}_{1}^{1}=x, \tilde{x}_{1}^{2}=z, \tilde{x}_{1}^{3}=y$. Put $\tilde{l}_{1}=l_{1}-2 y, \tilde{l}_{2}=l_{2}$ and $\tilde{l}_{3}=l_{1}-2 x$. It is easy to check that $\tilde{D}_{2} \tilde{D}_{1}\left(a_{\lambda}\right)=D_{2} D_{1}\left(a_{\lambda}\right)$ and deduce by arguments of Example 3.3 that the virtual polytope $\tilde{D}_{3} \tilde{D}_{2} \tilde{D}_{1}\left(a_{\lambda}\right)$ (see Figure 4 ) has the same character as the polytope $D_{1} D_{2} D_{1}\left(a_{\lambda}\right)$. In particular, the image of $\tilde{D}_{3} \tilde{D}_{2} \tilde{D}_{1}\left(a_{\lambda}\right)$ under the projection $(x, y, z) \mapsto(x+y, z)$ coincides with the weight polytope of the irreducible representation of $S L_{3}$ with the highest weight $-c \alpha_{1}-(a+b) \alpha_{2}$ (provided that the latter is dominant, that is, $a+b-2 c \geq 0$ and $c-2(a+b) \geq 0)$. The virtual polytope $\tilde{D}_{3} \tilde{D}_{2} \tilde{D}_{1}\left(a_{\lambda}\right)$ is a twisted cube of Grossberg-Karshon (cf. GK, Figure 2]) given by the inequalities

$$
a \leq x \leq c-2 b-a, \quad c \leq z \leq x+b-c, \quad b \leq y \leq-2 x+z-b .
$$

Note that the last pair of inequalities is inconsistent when $b>-2 x+z-b$, and should be interpreted in the sense of convex chains. More precisely, $\tilde{D}_{3} \tilde{D}_{2} \tilde{D}_{1}\left(a_{\lambda}\right)=\mathbb{I}_{P}-\mathbb{I}_{Q}$, where $P$ is the convex polytope given by inequalities $(G K)$ and $Q$ is the set given by the inequalities

$$
a \leq x \leq c-2 b-a, \quad c \leq z \leq x+b-c, \quad b>y>-2 x+z-b .
$$

(cf. [GK, Formula (2.21)]). A generalization of this example will be given in Section 4.3 .

\section{Bott towers And Bott-SAmelson Resolutions}

In this section, we outline possible algebro-geometric applications of the convexgeometric Demazure operators.

4.1. Bott towers. Let us recall the definition of a Bott tower (see GK] for more details). It is a toric variety obtained from a point by iterating the following step. Let $X$ be a toric variety, and $L$ a line bundle on $X$. Define a new toric variety $Y:=\mathbb{P}\left(L \oplus \mathcal{O}_{X}\right)$ as the projectivization of the split rank two vector bundle $L \oplus \mathcal{O}_{X}$ on $X$. Consider a sequence of toric varieties

$$
Y_{0} \leftarrow Y_{1} \leftarrow \ldots \leftarrow Y_{d}
$$




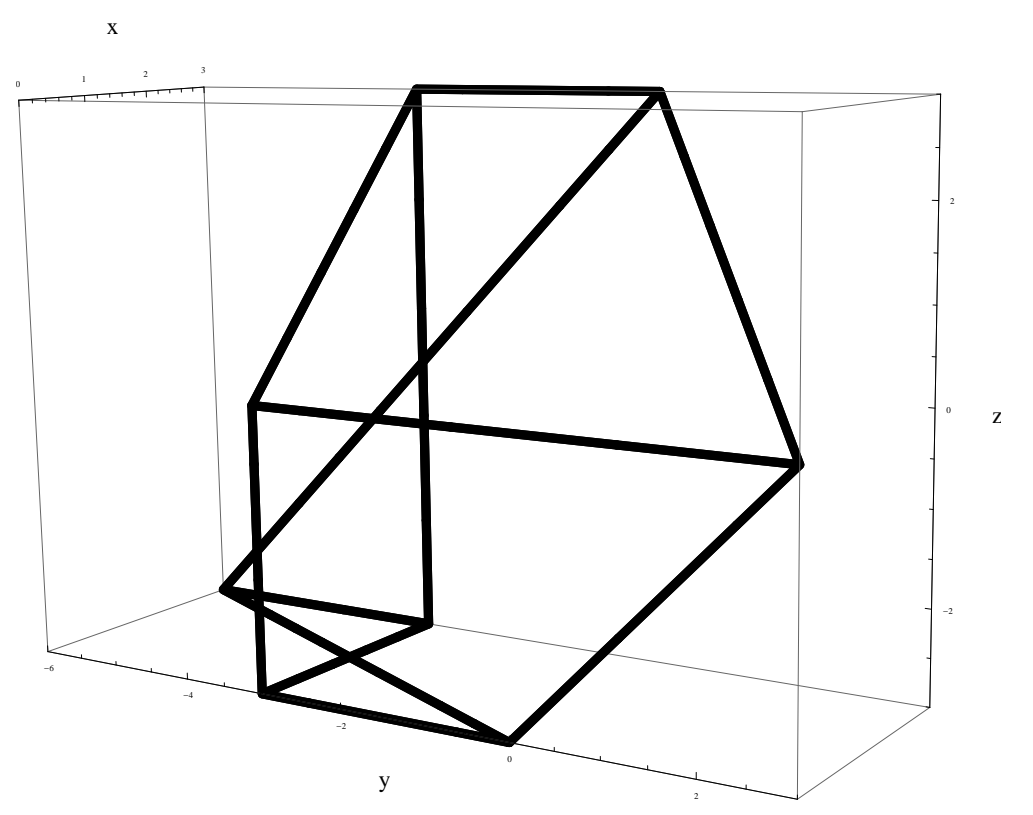

FiguRE 4. Virtual polytope $\tilde{D}_{3} \tilde{D}_{2} \tilde{D}_{1}\left(a_{\lambda}\right)$ for $a_{\lambda}=(0,-3,-3)$

where $Y_{0}$ is a point, and $Y_{i}=\mathbb{P}\left(L_{i-1} \oplus \mathcal{O}_{Y_{i-1}}\right)$ for a line bundle $L_{i-1}$ on $Y_{i-1}$. In particular, $Y_{1}=\mathbb{P}^{1}$ and $Y_{2}=\mathbb{P}\left(\mathcal{O}_{\mathbb{P}^{1}} \oplus \mathcal{O}_{\mathbb{P}^{1}}(k)\right)$ is a Hirzebruch surface. We call $Y_{d}$ the Bott tower corresponding to the collection of line bundles $\left(L_{1}, \ldots, L_{d-1}\right)$. Note that the collection $\left(L_{1}, \ldots, L_{d-1}\right)$ depends on $\frac{d(d-1)}{2}$ integer parameters since $\operatorname{Pic}\left(Y_{i}\right)=\mathbb{Z}^{i}$. Recall that the Picard group of a toric variety of dimension $d$ can be identified with a group of virtual lattice polytopes in $\mathbb{R}^{d}$ in such a way that very ample line bundles get identified with their Newton polytopes. One can describe the (possibly virtual) polytope $P(\mathcal{L})$ of a given line bundle $\mathcal{L}$ on $Y_{d}$ using a suitable string space.

Consider a string space with $d=r$, that is, $d_{1}=\ldots=d_{r}=1$. We have the decomposition

$$
\mathbb{R}^{d}=\underbrace{\mathbb{R} \oplus \ldots \oplus \mathbb{R}}_{d} .
$$

Label coordinates in $\mathbb{R}^{d}$ as follows: $x_{1}^{i}:=y_{i}$ for $i=1, \ldots, d$. Since we will be interested in the polytope $P:=D_{1} \ldots D_{d}(a)$, we can assume that the linear function $l_{i}$ for $i<d$ does not depend on $y_{1}, \ldots, y_{i}$, and $l_{d}=y_{1}$. Hence, the collection $\left(l_{1}, \ldots, l_{d-1}\right)$ of linear functions also depends on $\frac{d(d-1)}{2}$ parameters.

The projective bundle formula gives a natural basis $\left(\eta_{1}, \ldots, \eta_{d}\right)$ in the Picard group of $Y_{d}$. Namely, for $d=1$, the basis in $\operatorname{Pic}\left(\mathbb{P}^{1}\right)$ consists of the class of a point in $\mathbb{P}^{1}$. We now proceed by induction. Let $\left(\eta_{1}, \ldots, \eta_{i-1}\right)$ be the basis in $\operatorname{Pic}\left(Y_{i-1}\right)$ (we identify $\operatorname{Pic}\left(Y_{i-1}\right)$ with its pull-back to $\operatorname{Pic}\left(Y_{d}\right)$ ). Put $\eta_{i}=c_{1}\left(\mathcal{O}_{Y_{i}}(1)\right)$ where $c_{1}\left(\mathcal{O}_{Y_{i}}(1)\right)$ denotes the first Chern class of the tautological quotient line bundle 


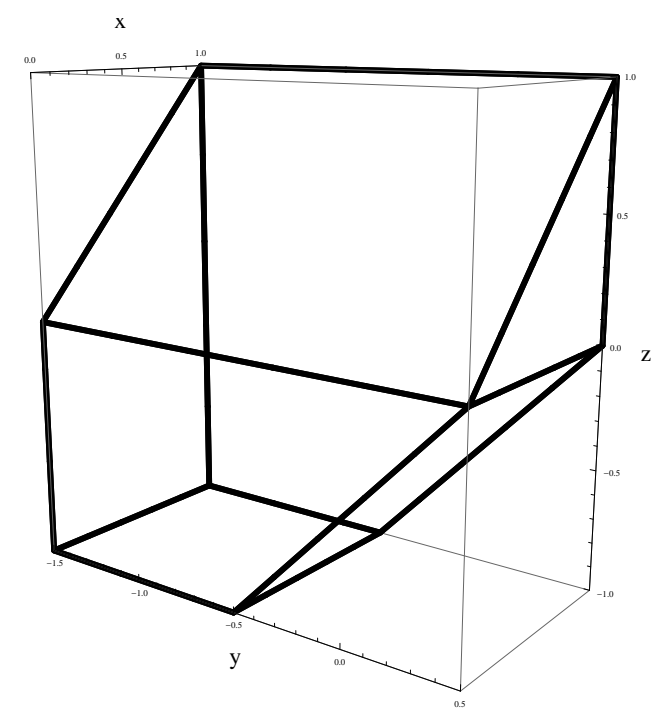

Figure 5. Polytope $D_{1} E_{u} D_{2} D_{1}(a)$ for $a=(0,-1,-1)$ and $u=(0,-1 / 2,0)$

$\mathcal{O}_{Y_{i}}(1)$ on $Y_{i}$. Decompose $L_{1}, \ldots, L_{d-1}$ in the basis $\left(\eta_{1}, \ldots, \eta_{d}\right)$ :

$$
L_{1}=a_{1,1} \eta_{1}, \quad \ldots, \quad L_{d-1}=a_{d-1,1} \eta_{1}+\ldots+a_{d-1, d-1} \eta_{d-1} .
$$

Similarly, decompose $l_{1}, \ldots, l_{d-1}$ in the basis of coordinate functions $\left(y_{1}, \ldots, y_{d}\right)$ :

$$
l_{1}=b_{1,1} y_{2}+\ldots+b_{1, d-1} y_{d}, \quad \ldots, \quad l_{d-1}=b_{d-1, d-1} y_{d} .
$$

Let $Y_{d}$ be the Bott tower corresponding to the collection $(*)$ of line bundles, and $\mathbb{R}^{d}$ the string space corresponding to the collection $(* *)$ of linear functions. One can show (cf. [GK, Theorem 3]) that if $a_{i, j}=b_{j, i}$, then there exists $a_{\mathcal{L}} \in \mathbb{R}^{d}$ such that

$$
P(\mathcal{L})=D_{1} D_{2} \ldots D_{d}\left(a_{\mathcal{L}}\right)
$$

In particular, when $\mathcal{L}$ is very ample the polytope $D_{1} D_{2} \ldots D_{d}\left(a_{\mathcal{L}}\right)$ coincides with the Newton polytope of the pair $\left(Y_{d}, \mathcal{L}\right)$. Note that the intermediate polytopes $\left\{a_{\mathcal{L}}\right\} \subset D_{d}\left(a_{\mathcal{L}}\right) \subset D_{d-1} D_{d}\left(a_{\mathcal{L}}\right) \subset \ldots$ correspond to the flag of toric subvarieties $Z_{0}=\{p t\} \subset Z_{1} \subset \ldots \subset Z_{d}=Y_{d}$, where $Z_{i}=p_{d-i}^{-1}\left(Z_{0}\right)$ and $p_{i}$ is the projection $p_{i}: Y_{d} \rightarrow Y_{i}$.

4.2. Bott-Samelson varieties. Similarly to Bott towers, Bott-Samelson varieties can be obtained by successive projectivizations of rank two vector bundles. In general, these bundles are no longer split, so the resulting varieties are not toric. In [GK], Bott-Samelson varieties were degenerated to Bott towers by changing complex structure (in particular, Bott-Samelson varieties are diffeomorphic to Bott towers when regarded as real manifolds). Below we define these varieties using notation of Section 3.3 .

Fix a Borel subgroup $B \subset G$. With every collection of simple roots $\left(\alpha_{i_{1}}, \ldots, \alpha_{i_{\ell}}\right)$, one can associate a Bott-Samelson variety $R_{\left(i_{1}, \ldots, i_{\ell}\right)}$ and a map $R_{\left(i_{1}, \ldots, i_{\ell}\right)} \rightarrow G / B$ by 
the following inductive procedure. Put $R_{\varnothing}=p t$. For every $\ell$-tuple $I=\left(i_{1}, \ldots, i_{\ell}\right)$ denote by $I^{j}$ the $(\ell-1)$-tuple $\left(i_{1}, \ldots, \hat{i}_{j}, \ldots, i_{\ell}\right)$. Define $R_{I}$ as the fiber product $R_{I^{\ell}} \times_{G / P_{\ell}} G / B$, where $P_{i_{\ell}}$ is the minimal parabolic subgroup corresponding to the root $\alpha_{i_{\ell}}$. The map $r_{I}: R_{I} \rightarrow G / B$ is defined as the projection to the second factor. There is a natural embedding

$$
R_{I^{\ell}} \hookrightarrow R_{I} ; \quad x \mapsto\left(x, r_{I}(x)\right) .
$$

In particular, any subsequence $J \subset I$ yields the embedding $R_{J} \hookrightarrow R_{I}$.

It follows from the projective bundle formula that the Picard group of $R_{I}$ is freely generated by the divisors $R_{I^{1}}, \ldots, R_{I^{\ell}}$. Denote by $v$ the geometric valuation on $\mathbb{C}\left(R_{I}\right)$ defined by the flag $R_{\varnothing} \subset R_{\left(i_{\ell}\right)} \subset R_{\left(i_{\ell-1}, i_{\ell}\right)} \subset \ldots \subset R_{I^{1}} \subset R_{I}$. Let $\mathcal{L}$ be a line bundle on $R_{I}$, and $P_{v}(\mathcal{L})$ its Okounkov body with respect to the valuation $v$. Conjecturally, $P_{v}(\mathcal{L})$ can be described using string spaces as follows.

Replace a reduced decomposition of $w_{0}$ in the definition of the string space from Section 3.3 by a sequence $\left(\alpha_{i_{1}}, \ldots, \alpha_{i_{\ell}}\right)$ that defines $R_{I}$ (we no longer require that $s_{i_{1}} \ldots s_{i_{\ell}}$ be reduced). More precisely, let $d_{i}$ the number of $\alpha_{i_{j}}$ in this sequence such that $i_{j}=i$. We get the following string space $\mathcal{S}_{I}$ of rank $\leq r$ and dimension $\ell$ :

$$
\mathbb{R}^{\ell}=\mathbb{R}^{d_{1}} \oplus \ldots \oplus \mathbb{R}^{d_{r}}
$$

where the functions $l_{i}$ are given by the formula:

$$
l_{i}(x)=\sum_{k \neq i}\left(\alpha_{k}, \alpha_{i}\right) \sigma_{k}(x) .
$$

In particular, if $\ell=d$ and $s_{i_{1}} \cdots s_{i_{\ell}}$ is reduced then $R_{I}$ is a Bott-Samelson resolution of the flag variety $G / B$, and $\mathbb{R}^{\ell}$ is exactly the string space from Section 3.3 . Denote by $E_{u}$ the parallel translation in the string space by a vector $u \in \mathbb{R}^{\ell}$.

Conjecture 4.1. For every line bundle $\mathcal{L}$ on $R_{I}$, there exists a point $\mu \in \mathbb{R}^{r}$ and vectors $u_{1}, \ldots, u_{\ell} \in \mathbb{R}^{\ell}$ such that we have

$$
P(\mathcal{L})=E_{u_{1}} D_{i_{1}} E_{u_{2}} D_{i_{2}} \ldots E_{u_{\ell}} D_{i_{\ell}}\left(a_{\mu}\right)
$$

for any point $a_{\mu} \in \mathbb{R}^{\ell}$ that satisfies $p(a)=\mu$.

In particular, if $\mathcal{L}=r_{I}^{*} \mathcal{L}(\lambda)$, where $\mathcal{L}(\lambda)$ is the line bundle on $G / B$ corresponding to the dominant weight $\lambda$, then one can take $u_{2}=\ldots=u_{\ell}=0$ and $\mu=\lambda$. This conjecture agrees with the example computed in [Anderson, Section 6.4] for $S L_{3}$ and the Bott-Samelson resolution $R_{(1,2,1)}$ (cf. Figure 5 and Figure 3(b) in loc.cit.). Figure 5 shows the polytope $D_{1} E_{u} D_{2} D_{1}(a)$ for the string space $(B S)$ when $G=S L_{3}$ and $I=(1,2,1)$.

4.3. Degenerations of string spaces. While twisted cubes of Grossberg-Karshon for $G L_{n}$ and Gelfand-Zetlin polytopes have different combinatorics they produce the same Demazure characters. We now reproduce this phenomenon for general string spaces. In particular, we transform the string space $(B S)$ from Section 4.2 into a string space from Section 4.1. 
Let $\mathcal{S}$ be a string space $\mathbb{R}^{d}=\mathbb{R}^{d_{1}} \oplus \ldots \oplus \mathbb{R}^{d_{r}}$ with functions $l_{1}, \ldots, l_{r}$. Suppose that $d_{i}>1$.

Definition 4 . The $i$-th degeneration of the string space $\mathcal{S}$ is the string space

$$
\mathbb{R}^{d}=\mathbb{R}^{d_{1}} \oplus \ldots \oplus \underbrace{\mathbb{R}^{d_{i}-1} \oplus \mathbb{R}^{1}}_{\mathbb{R}^{d_{i}}} \oplus \ldots \oplus \mathbb{R}^{d_{r}}
$$

of rank $(r+1)$ with functions $l_{1}, \ldots, l_{i}^{\prime}, l_{i}^{\prime \prime}, \ldots, l_{r}$, where

$$
l_{i}^{\prime}(x)=l_{i}(x)-2 x_{d_{i}}^{i} ; \quad l_{i}^{\prime \prime}(x)=l_{i}(x)-2 \sum_{k=1}^{d_{i}-1} x_{k}^{i} .
$$

Example 4.2. The string space $\mathbb{R} \oplus \mathbb{R} \oplus \mathbb{R}$ from Example 3.4 is the 1-st degeneration of the space $\mathbb{R}^{2} \oplus \mathbb{R}$ with $l_{1}=x_{1}^{1}+x_{2}^{1}, l_{2}=x_{1}^{2}$.

Define the projection $p_{i}: \mathbb{R}^{r+1} \rightarrow \mathbb{R}^{r}$ by sending $\left(y_{1}, \ldots, y_{i}^{\prime}, y_{i}^{\prime \prime}, \ldots, y_{r}\right)$ to $\left(y_{1}, \ldots, y_{i}^{\prime}+y_{i}^{\prime \prime}, \ldots, y_{r}\right)$. This projection induces a homomorphism of group algebras of the lattices $\mathbb{Z}^{r+1}$ and $\mathbb{Z}^{r}$, which we will also denote by $p_{i}$. It is easy to check that

$$
T_{i} \circ p_{i}=p_{i} \circ T_{i}^{\prime}=p_{i} \circ T_{i}^{\prime \prime} .
$$

Combining this observation with Theorem 3.2 , we get the following proposition.

Proposition 4.3. For any polytope $P \subset \mathbb{R}^{d}$ such that $\operatorname{dim} P \cap \mathbb{R}^{d_{i}}<d_{i}$, we have

$$
\chi\left(D_{i}(P)\right)=p_{i}\left(\chi\left(D_{i}^{\prime}(P)\right)\right)=p_{i}\left(\chi\left(D_{i}^{\prime \prime}(P)\right)\right) .
$$

We now degenerate successively the string spaces from Section 4.2 , Let $I=$ $\left(\alpha_{i_{1}}, \ldots, \alpha_{i_{\ell}}\right)$ be a sequence of simple roots, and

$$
\mathbb{R}^{\ell}=\mathbb{R}^{d_{1}} \oplus \ldots \oplus \mathbb{R}^{d_{r}}
$$

is the corresponding string space $\mathcal{S}_{I}$ with the functions $l_{1}, \ldots, l_{r}$ given by $(B S)$.

Let $\tilde{\mathcal{S}}_{I}$ be the string space of rank $\ell$ obtained from $S_{I}$ by $\left(d_{1}-1\right)$ first degenerations, $\left(d_{2}-1\right)$ second degenerations etc., that is,

$$
\tilde{\mathcal{S}}_{I}=\underbrace{\mathbb{R}_{1}^{(1)} \oplus \ldots \oplus \mathbb{R}_{d_{1}}^{(1)}}_{\mathbb{R}^{d_{1}}} \oplus \ldots \oplus \underbrace{\mathbb{R}_{1}^{(\ell)} \oplus \ldots \oplus \mathbb{R}_{d_{\ell}}^{(\ell)}}_{\mathbb{R}^{d_{\ell}}},
$$

where the functions $l_{j}^{(i)}$ are given by the formula:

$$
l_{j}^{(i)}(x)=l_{i}(x)-2 \sum_{k \neq j} x_{k}^{i} .
$$

Denote the Demazure operators associated with $\tilde{\mathcal{S}}_{I}$ by $\tilde{D}_{j}^{(i)}$.

For a point $a \in \mathbb{R}^{\ell}$, consider the convex chain $P_{I}=D_{i_{1}} \ldots D_{i_{\ell}}(a)$. For every $k=1, \ldots, r$, we now replace the rightmost $D_{k}$ in the expression $D_{i_{1}} \ldots D_{i_{\ell}}$ by $D_{1}^{(k)}$, the next one by $D_{2}^{(k)}, \ldots$, the leftmost by $D_{i_{k}}^{(k)}$. Denote the resulting convex chain by $\tilde{P}_{I}$. 
Example 4.4. If $r=3, \ell=6$ and $I=\left(\alpha_{1}, \alpha_{2}, \alpha_{1}, \alpha_{3}, \alpha_{2}, \alpha_{1}\right)$, then

$$
P_{I}=D_{1} D_{2} D_{1} D_{3} D_{2} D_{1}, \quad \tilde{P}_{I}=\tilde{D}_{3}^{(1)} \tilde{D}_{2}^{(2)} \tilde{D}_{2}^{(1)} \tilde{D}_{1}^{(3)} \tilde{D}_{1}^{(2)} \tilde{D}_{1}^{(1)}(a) .
$$

Proposition 4.3 implies that $P_{I}$ and $\tilde{P}_{I}$ have the same character (with respect to the map $\left.p: x \mapsto \sigma_{1}(x) \alpha_{1}+\ldots+\sigma_{r}(x) \alpha_{r}\right)$.

Corollary 4.5. If $p(a)$ is dominant and $s_{i_{1}} \cdots s_{i_{\ell}}$ is reduced, then the corresponding Demazure character coincides with $\chi\left(P_{I}\right)=\chi\left(\tilde{P}_{I}\right)$.

The proof is completely analogous to the proof of Theorem 3.6.

Remark 4.6. Note that the convex chain $\tilde{P}_{I}$ coincides with the twisted cube constructed in [GK] for the corresponding Bott-Samelson resolution. Indeed, $\left(\alpha_{i}, \alpha_{i}\right)=$ -2 according to our definition of the function $\left(\cdot, \alpha_{i}\right)$ (see Section 3.3), hence, we can write

$$
l_{j}^{(i)}(x)=\sum_{(p, q) \neq(i, j)}\left(\alpha_{p}, \alpha_{i}\right) x_{q}^{p} .
$$

It is now easy to check that the defining inequalities for $\tilde{P}_{I}$ coincide with the inequalities given by [GK, Formula (2.21)] together with computations of [GK, Section 3.7].

The string space $\mathcal{S}_{I}$ and its complete degeneration $\tilde{\mathcal{S}}_{I}$ are two extreme cases that yield convex chains for given Demazure characters. By taking partial degenerations of $\mathcal{S}_{I}$ one can construct intermediate convex chains with the same character. However, only $\mathcal{S}_{I}$ might produce true polytopes (such as Gelfand-Zetlin polytopes for $G=S L_{n}$ or polytope of Example 3.4 for $G=S p_{4}$ ) that represent the Weyl characters. Indeed, such a polytope must have a face $D_{i}^{2}(a)$ (in the case of $\mathcal{S}_{I}$ ) or a face $D_{i}^{\prime} D_{i}^{\prime \prime}(a)$ (in the case of the $i$-th degeneration of $\mathcal{S}_{I}$ ). The former is a true segment since $D_{i}^{2}=D_{i}$, while the latter is necessarily a virtual trapezoid with the same character due to cancelations (cf. Figure 3).

\section{REFERENCES}

[Andersen] H. H. Andersen, Schubert varieties and Demazure's character formula, Invent. Math., 79 (1985), no. 3, 611-618

[Anderson] D. Anderson, Okounkov bodies and toric degenerations, preprint arXiv:1001.4566v3 [math.AG], to appear in Math. Ann.

[BZ] A.Berenstein, A. Zelevinsky, Tensor product multiplicities, canonical bases and totally positive varieties, Invent. Math. 143 (2001), no.1, 77-128

[GK] M. Grossberg and Y. Karshon, Bott towers, complete integrability, and the extended character of representations, Duke Math. J., 76 (1994), no. 1, 23-58.

[K] K.KaveH, Crystal basis and Newton-Okounkov bodies, preprint arXiv:1101.1687 [math.AG]

[KKh] K. Kaveh, A. KhovanskiI, Convex bodies associated to actions of reductive groups, Moscow Math.J., 12 (2012), 369-396

[PKh] A.G. Khovanskit, A.V. Pukhlikov, Finitely additive measures of virtual polytopes, St. Petersburg Mathematical Journal, 4 (1993), no.2, 337-356 
[PKh2] A.G.Khovanskil and A.V.Pukhlikov, The Riemann-Roch theorem for integrals and sums of quasipolynomials on virtual polytopes, Algebra i Analiz, 4 (1992), no.4, 188-216

[KST] V. Kiritchenko, E. Smirnov, V. Timorin, Schubert calculus and Gelfand-Zetlin polytopes, Russian Math. Surveys, 67 (2012), no.4, 685-719

[L] P. Littelmann, Cones, crystals and patterns, Transformation Groups, 3 (1998), pp. 145179

[KnM] A. Knutson And E. Miller, Gröbner geometry of Schubert polynomials, Ann. of Math. (2), 161 (2005), 1245-1318

E-mail address: vkiritch@hse.ru

Laboratory of Algebraic Geometry and Faculty of Mathematics, Higher School of Economics, Vavilova St. 7, 112312 Moscow, Russia

Institute for Information Transmission Problems, Moscow, Russia 